\title{
Conceptos básicos sobre la formación y teoría de los vórtices
}

\author{
H. Gardea-Villegas \\ Profesor de la Facultad de Ingeniería, UNAM \\ E-mail:gardea@servidor.unam.mx \\ (recibido: abril de 1999; aceptado: febrero de 2001)
}

\begin{abstract}
Resumen
Los vórtices son un fenómeno cotidiano que tienen una importancia especial en la hidráulica, pero no puede decirse que sean de su exclusiva competencia. En meteorología, los vórtices son los ciclones o remolinos que estamos acostumbrados a ver desde siempre; sin embargo, el estudiante de ingeniería se enfrenta ante estos fenómenos hasta que decide cursar el posgrado en hidráulica. Este hecho, hace creer que se trata de un tema especialmente complicado, por lo que deja a la mayor parte de los ingenieros civiles fuera de este conocimiento. Este desinterés perjudica el desarrollo profesional debido a que prácticamente no hay obra hidráulica donde no aparezcan vórtices y sus efectos, que en el flujo, son generalmente importantes.

Este artículo presenta de forma accesible para el ingeniero, la teoría de los dos tipos de vórtices que se conocen. Para disponer de apoyo matemático, se hace una retrospectiva de las herramientas necesarias que permiten comprender la teoría, tanto de los vórtices libres como de los forzados. También se demuestra por qué un vórtice puede ser rotacional o irrotacional. Finalmente, el autor pretende que estos conocimientos básicos sean de utilidad para quien desee adentrarse en el tema o, incluso hacer sus propias investigaciones.
\end{abstract}

Descriptores: vórtice, flujo, ciclón, rotacional, circulación.

Abstract

Vortices constitute a phenomenon of very frequent occurrence, and although they are especially important in hydraulics, we may agree that they are not of the exclusive realm of this field. For example, in meteorology, cyclones or eddies are vortices that everyone has witnessed oftentimes in a life time. Nevertheless, a civil engineering student is aware about them only when he decides to pursue graduate studies. This fact leads us to assume that the study of a vortex is a very difficult subject or that perhaps it is considered unimportant for a non-specialized engineer. If we notice however that almost in every water flow a vortex appears, we should seriously think about the convenience of learning more about the problem; therefore we should become familiar with this topic in due time. That is particularly valid for engineers dealing with hydraulic projects.

The purpose of this paper is to show, in an accessible approach for a non-specialized civil engineer, the theory of the two main types of vortices known (free vortex and forced vortex). Furthermore, in order to better understand the theory, mathematical aids needed to explain it are included. There is also a stepby-step demonstration of the fact that a vortex can be rotational or irrotational and that the phenomenon depends on different laws. With that knowledge, the engineer presumably will be able to deal with the problems concerning vortices. It is also believed that this paper would help the engineer to get acquainted with the problem and, maybe, to feel more motivated to perform his own research. 


\section{Introducción}

En su clasificación más general, puede decirse que existen dos tipos de vórtices a saber: el vórtice forzado y el vórtice libre.

El vórtice forzado es el más fácil de entender, ya que se genera por el contacto entre un fluido real en movimiento y un obstáculo que puede ser una pared fija u otro fluido cuya velocidad sea contraria a la del primero, o simplemente cuya velocidad sea sensiblemente distinta a la del mencionado fluido real. Este caso se presenta cuando una corriente de agua se aproxima a una transición o a cualquier obstáculo que debido al contacto con este cuerpo sólido, genera una rotación que provoca la formación del fenómeno. Estos vórtices son superficiales y no tienen salida por su parte inferior como los vórtices libres, en realidad, se trata de depresiones cóncavas que giran en la superficie del agua y su sentido de giro es el que imprime el choque del agua con el obstáculo mencionado. En la figura 1 se indican dos casos de vórtices forzados.

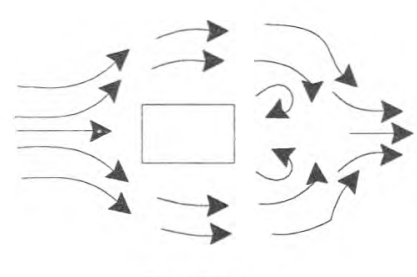

a) obstáculo

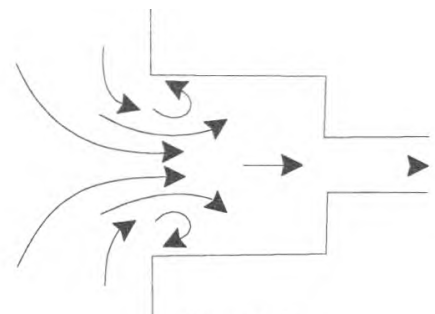

b) transición
Figura 1. Vórtices forzados

El vórtice libre se presenta típicamente sobre una descarga que puede ser "de fondo" como sucede en las tinas de baño o en los lavamanos al vaciarse, cuando el líquido se encontraba originalmente en reposo. Un caso semejante es el del vórtice que se forma debido a la succión provocada en un tanque por la tubería de succión de una bomba, o bien, el que se presenta en las obras de toma de las presas.

Para que se forme un vórtice libre debe existir un punto de baja presión que succione el flujo hacia él. Este punto llamado núcleo, es el que en la atmósfera produce un gradiente de presiones que hace que los vientos provenientes de zonas con mayor presión se dirijan hacia él y formen los vórtices que comúnmente llamamos remolinos, o cuando es mayor su magnitud ciclones o tornados.

En el caso de los líquidos, la depresión en el núcleo se debe a la succión ya mencionada o a cualquier otro fenómeno físico que produzca una sensible caída de presión. Un ejemplo de lo antes descrito fue lo que observó el Dr. Gabriel Echávez, quien al colaborar con el Dr. Enzo-Levi (1989) en sus investigaciones en el Instituto de Ingeniería de la UNAM, descubrió que si en un recipiente que contenga un líquido en reposo, se inyecta bajo el agua con un chorro inclinado y orientado hacia el fondo, también aparece un vórtice en la superficie del tanque. La formación de este vórtice se ajusta a lo que se mencionó anteriormente, ya que la corriente concentrada bajo el agua dentro de un medio estático, genera necesariamente una caída de presión a su alrededor, creando así el núcleo del vórtice. En realidad no se sabe con precisión a qué se debe la formación de un vórtice libre pero es un hecho que existe, y como sucede con otros fenómenos físicos cuya existencia no podríamos intuir sin haberlos visto antes, ha sido posible desarrollar una fundamentación matemática que los explique. En la figura 2 se representan tres ejemplos de este tipo de vórtice.

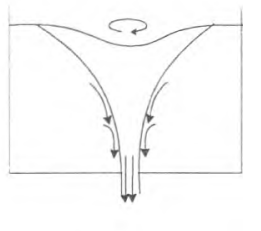

a) descarga de fondo

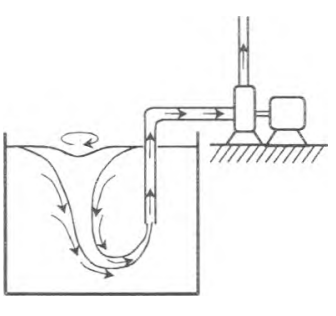

b) succión de una bomba

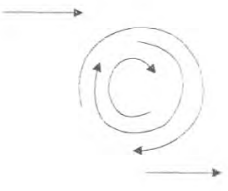

c) ałmósfera
Figura 2. Vórtices libres

\section{Sentido de giro de los vórtices libres}

El sentido del giro de este tipo de vórtices depende del hemisferio terrestre en que se encuentren. Si nos ubicamos en cualquiera de los puntos $\mathrm{O}$ de baja presión que se indican en la figura 3, existe un gradiente de presiones que dirige el flujo de los puntos $\mathrm{A}$ y $\mathrm{B}$ hacia el núcleo $\mathrm{O}$.

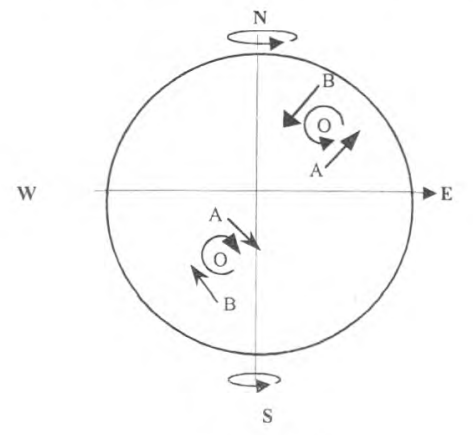

Figura 3 
Debido a la rotación (antihoraria) de la tierra, la velocidad tangencial original de los puntos $\mathrm{A}$ es mayor que la de los puntos $B$, ya que por encontrarse los primeros en zonas de menor latitud tienen un radio terrestre mayor (medido en un plano paralelo al ecuador). Por este motivo, al dirigirse un fluido de $\mathrm{A}$ hacia $\mathrm{O}$, animado con mayor velocidad tangencial que el que se encuentra en $\mathrm{O}$, este último se queda atrás de A ( por decirlo en esta forma) produciéndose un desvío de la corriente en el hemisferio norte hacia la derecha y en el hemisferio sur hacia la izquierda. Un razonamiento semejante, lleva a la conclusión de que los puntos $\mathrm{B}$ tienen la misma tendencia. Al acercarse el fluido al núcleo que lo atrae, gira en forma indicada en la figura 3, por esta razón, se forman vórtices con sentido antihorario en el hemisferio norte y con sentido horario en el hemisferio sur, que son los sentidos de giro de los ciclones observados en la atmósfera.

Desde luego, tanto el vórtice forzado como el libre, están sujetos a leyes distintas, mismas que se explicarán después de hacer una introducción a las herramientas matemáticas necesarias para el estudio de estos fenómenos.

\section{Conceptos matemáticos}

Operador "nabla"

Se llama así el siguiente concepto:

$$
\nabla=\mathbf{i} \frac{\partial}{\partial \mathbf{x}}+\mathbf{j} \frac{\partial}{\partial \mathrm{y}}+\mathbf{k} \frac{\partial}{\partial z}
$$

En que $\boldsymbol{i}, \boldsymbol{j}$ y $\boldsymbol{k}$ son los vectores unitarios en los sentidos de los ejes coordenados $\mathbf{X}, \mathbf{Y}$ y $\mathbf{Z}$, respectivamente.

Rotacional de un vector

El rotacional de un vector $\mathrm{V}$ es el producto vectorial del operador $\nabla$ por dicho vector. Es decir, se trata del vector:

$$
\operatorname{rot} \mathrm{V}=\nabla \times \mathrm{V}
$$

Si $\mathrm{V}=i u+j v+k w$, en que $u, v$ y $w$ son respectivamente las proyecciones del módulo del vector $\mathrm{V}$ sobre los ejes $\mathrm{X}, \mathrm{Y}$ y Z $\mathrm{Z}$, el producto vectorial anterior equivale a:

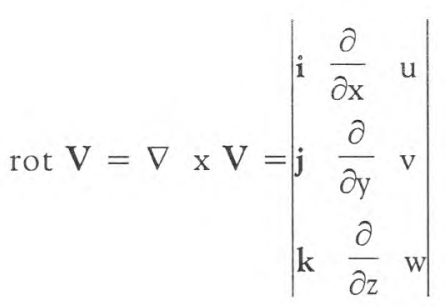

$$
\operatorname{rot} V=i\left(\frac{\partial w}{\partial y}-\frac{\partial v}{\partial z}\right)+j\left(\frac{\partial u}{\partial z}-\frac{\partial w}{\partial x}\right)+k\left(\frac{\partial v}{\partial x}-\frac{\partial u}{\partial y}\right)
$$

Consideremos ahora, una partícula diferencial que se desplaza siguiendo una trayectoria cualquiera, pero que gira al mismo tiempo con respecto a un eje perpendicular a dicha trayectoria. Si la vemos en el plano $\mathrm{X}-\mathrm{Y}$, quedando el eje $\mathbf{Z}$ como eje de giro, se puede representar en la figura 4a. Asimismo, las figuras $4 \mathrm{~b}$ y $4 \mathrm{c}$ corresponden a los otros planos del movimiento (indicados en las figuras respectivas).

En estas figuras se representa la partícula elemental con línea llena, y con línea interrumpida la misma partícula después de un tiempo dt en que ha sufrido un giro diferencial.
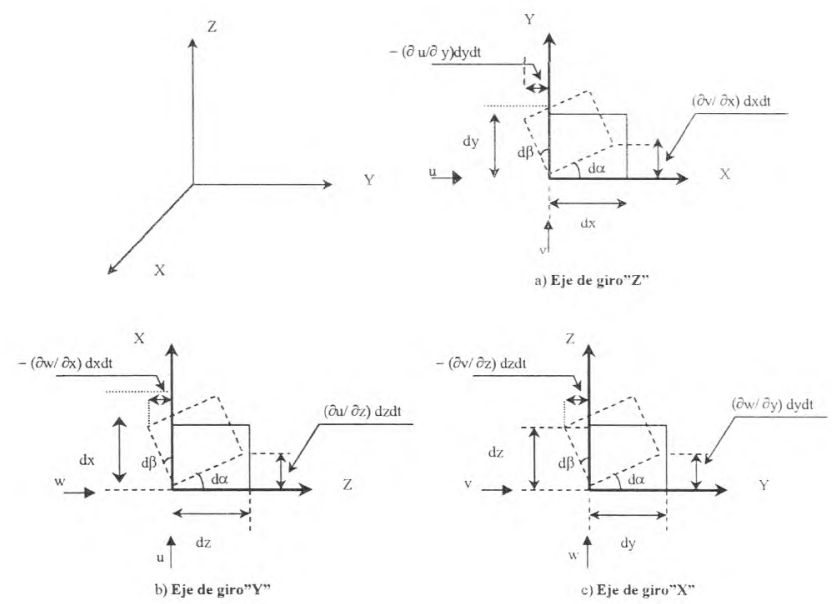

\section{Figura 4}

En cada caso, los ángulos $\mathrm{d} \alpha$ y $\mathrm{d} \beta$ pueden representarse en radianes. Por ejemplo, para el caso de la figura 4a ( plano $\mathrm{X}-\mathrm{Y})$.

$$
d \alpha=\frac{\frac{\partial v}{\partial x} d x \cdot d t}{d x}=\frac{\partial v}{\partial x} d t \quad \text { y análogamente } \quad d \beta=-\frac{\partial u}{\partial y} d t
$$

En forma semejante se pueden obtener los ángulos diferenciales de giro para los demás casos.

Si ahora llamamos vector torbellino a:

$$
\omega=i \omega_{x}+j \omega_{y}+k \omega_{z}
$$


En que $\omega_{x}, \omega_{y}$ y $\omega_{z}$ son en realidad los módulos del vector que representa la velocidad angular, proyectados en los planos:

$$
\mathrm{Y}-\mathrm{Z}\left(\omega_{x}\right) ; \mathrm{Z}-\mathrm{X}\left(\omega_{\mathrm{y}}\right) \text { y } \mathrm{X}-\mathrm{Y}\left(\omega_{z}\right)
$$

Ahora bien, como se sabe la velocidad angular es por definición:

$\omega=\frac{d \theta}{d t}$, en que $\theta$ es el ángulo de giro de una partícula en movimiento curvilíneo. Se tendrá entonces que el promedio de cada giro vale:

Para 4a:

$$
\omega_{z}=\frac{1}{2}\left(\frac{d \alpha}{d t}+\frac{d \beta}{d t}\right)=\frac{1}{2}\left(\frac{\partial v}{\partial x}-\frac{\partial u}{\partial y}\right)
$$

Para 4b:

$$
\omega_{y}=\frac{1}{2}\left(\frac{d \alpha}{d t}+\frac{d \beta}{d t}\right)=\frac{1}{2}\left(\frac{\partial u}{\partial z}-\frac{\partial w}{\partial x}\right)
$$

Para $4 c$ :

$$
\omega_{x}=\frac{1}{2}\left(\frac{d \alpha}{d t}+\frac{d \beta}{d t}\right)=\frac{1}{2}\left(\frac{\partial w}{\partial y}-\frac{\partial v}{\partial z}\right)
$$

Que según en el texto de Comolet (1963), son las componentes del vector:

$$
\frac{1}{2} \operatorname{rot} \mathrm{V}
$$

Esto significa que el vector torbellino, definido antes, tiene el siguiente valor:

$$
\omega=\frac{1}{2} \operatorname{rot} V
$$

Entonces, la condición para que la partícula gire es que exista $\omega$. En este caso, se habla de un flujo rotacional. Inversamente el flujo es irrotacional si:

$$
\operatorname{rot} \mathrm{V}=0
$$

\section{Circulación}

Se llama circulación $\Gamma$ a la integral lineal cerrada de la velocidad $\mathrm{V}$ en cada punto de una línea cerrada $\mathrm{L}$ en que
$\mathrm{dL}$ forma un ángulo $\theta$ con el vector $\mathrm{V}$ en cada punto de la línea. Es decir:

$$
\Gamma=\oint_{L} V d L
$$

Si nos referimos a una serie de líneas de corriente por simplicidad alojadas en un plano, la circulación $\Gamma$ de $\mathrm{V}$ a lo largo de una línea cerrada cualquiera alojada sobre las líneas de corriente de acuerdo con la figura 5, puede escribirse:

$$
\Gamma=\oint V \cos \theta \cdot d L
$$

(en términos escalares)

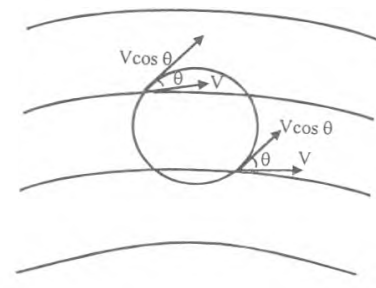

Figura 5

La circulación alrededor del elemento diferencial indicado en la figura 6 puede calcularse, como propone Daugherty (1985), tomando los promedios de las velocidades en cada esquina de dicho elemento (despreciando diferenciales de segundo orden).

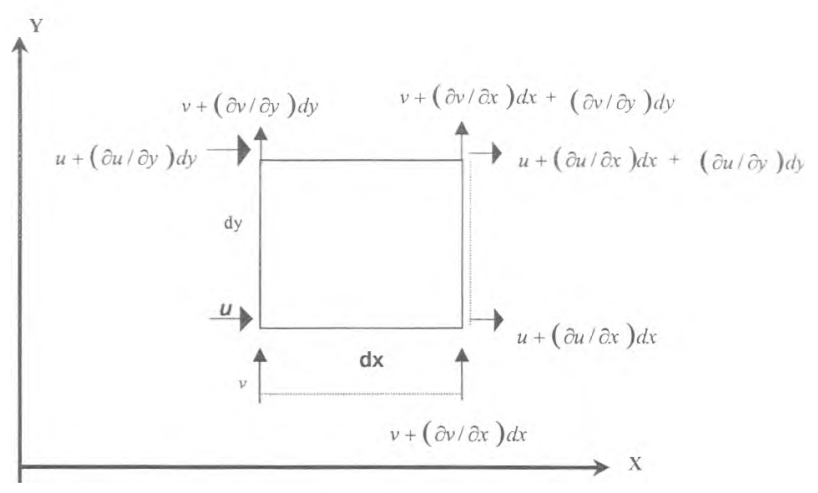

Figura 6

La circulación en sentido inverso (contrario a las manecillas del reloj) tiene el valor:

$$
\mathrm{d} \Gamma=\frac{1}{2}\left(u+u+\frac{\partial u}{\partial x} d x\right) d x+\frac{1}{2}\left(v+\frac{\partial v}{\partial x} d x+v+\frac{\partial v}{\partial x} d x+\frac{\partial v}{\partial y} d y\right) d y
$$




$$
\begin{aligned}
& -\frac{1}{2}\left(u+\frac{\partial u}{\partial x} d x+\frac{\partial u}{\partial y} d y+u+\frac{\partial u}{\partial y} d y\right) d x \\
& -\frac{1}{2}\left(v+\frac{\partial v}{\partial y} d y+v\right) d y=\left(\frac{\partial v}{\partial x}-\frac{\partial u}{\partial y}\right) d x d y
\end{aligned}
$$

Se llama vorticidad $\xi$ al cociente de la circulación alrededor de una línea cerrada entre el área que ésta contiene. Es decir, para el caso de la figura 6:

$$
\xi=\frac{d \Gamma}{d x d y}=\frac{\partial v}{\partial x}-\frac{\partial u}{\partial y}
$$

Observando la expresión (1) se concluye que otra forma de caracterizar un flujo irrotacional es señalar que en él la vorticidad es nula, es decir:

$$
\xi=0
$$

\section{Flujos irrotacional y rotacional}

En la figura 7, Kolar (1983), describe con gran claridad estos tipos de flujo. En efecto, si pensamos en una partícula de fluido desplazándose a lo largo de una trayectoria S, se dice que está sujeta a un flujo rotacional si, además de su movimiento según $\mathrm{S}$, gira sobre un eje normal a dicha trayectoria. Este es el caso representado en la figura $7 b$. Por otra parte, en la figura 7 a se ve un escurrimiento en que las partículas no cambian su orientación respecto a la dirección del flujo durante toda su trayectoria. En este último caso, se dice que se trata de un flujo irrotacional.

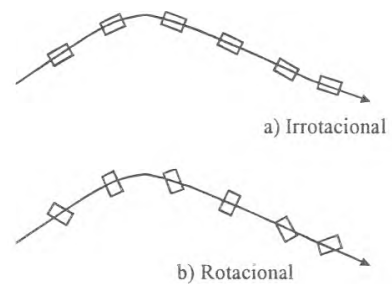

Figura 7

\section{Vórtice forzado}

En este caso, al encontrarse el flujo con un agente que lo haga girar, aparece una velocidad angular: $\omega=\mathrm{V} / \mathrm{r}$ en que $\mathrm{V}$ es la velocidad tangencial a la trayectoria y $\mathbf{r}$ el radio vector de giro de la partícula. Si se desprecia la fricción, puede considerarse que $\omega$ permanecerá constante.
Supongamos una partícula $\mathrm{P}(\mathrm{x}, \mathrm{y})$ que se encuentra girando con velocidad angular $\omega$, tal como se indica en la figura 8 .

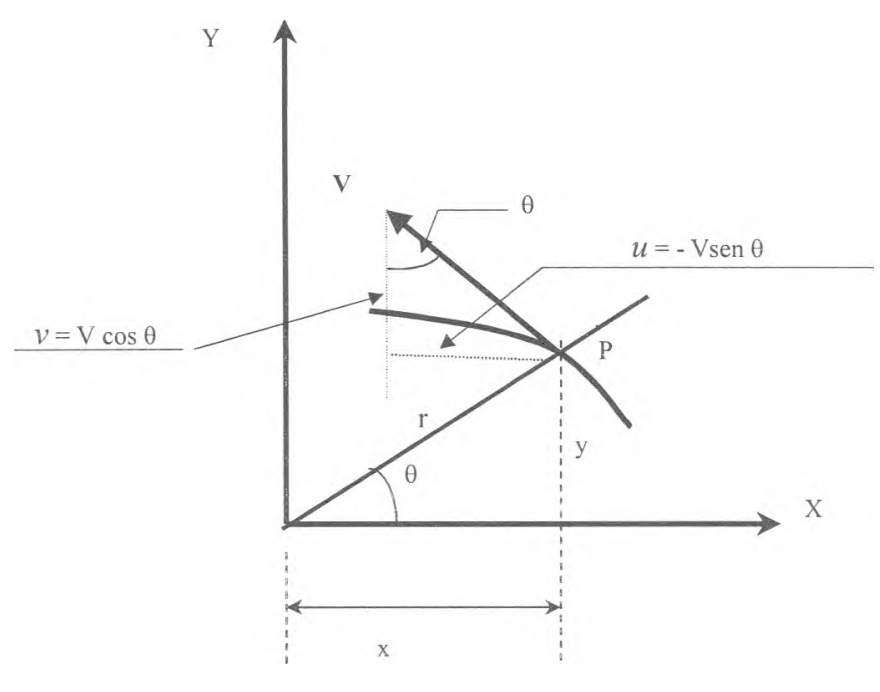

Figura 8

Como el $\operatorname{sen} \theta=y / r$ y el $\cos \theta=x / r$, de acuerdo con la figura 8 , y recordando que $\omega=V / r$, puede escribirse:

$$
\left.\begin{array}{l}
\mathrm{v}=\mathrm{V} \cos \theta=V \frac{x}{r}=\omega_{z} x \\
u=-V \operatorname{sen} \theta=-V \frac{\mathrm{y}}{\mathrm{r}}=-\omega_{z} y
\end{array}\right]
$$

Luego:

$$
\frac{\partial v}{\partial x}=\omega_{z} \quad y \quad \frac{\partial u}{\partial y}=-\omega_{z}
$$

Por lo que

$$
\frac{\partial v}{\partial x}-\frac{\partial u}{\partial y}=2 \omega_{z}
$$

Y análogamente

$$
\frac{\partial u}{\partial z}-\frac{\partial w}{\partial x}=2 \omega_{y} \quad y \quad \frac{\partial w}{\partial y}-\frac{\partial v}{\partial z}=2 \omega_{x}
$$

Es decir, el vector torbellino, según la definición vista en rotacional de un vector y la expresión (1), tiene el valor:

$$
\omega=\frac{1}{2} \operatorname{rot} \mathrm{V}
$$

Y como en este caso rot $V \neq 0$, al serlo sus componentes, significa que un vórtice forzado es rotacional. Lo mismo puede concluirse por el sólo hecho de que el vector torbellino existe.

Este tipo de vórtice es muy común. Tal es el caso de los que se forman cuando el flujo toca una pared rígida en 
una transición; por ejemplo, los formados atrás de los remos de una lancha semejantes a los que se forman al mover una cuchara dentro de una taza de café, o los vórtices alternos, llamados de "Von Kármán ", por ser él quien los estudió primero y que son los que se forman detrás de los barcos en movimiento y contribuyen a producir sus estelas.

\section{Vórtice libre}

En este caso, debemos suponer que el vórtice ya existe, cosa que la experiencia nos demuestra ampliamente. Ahora, con apoyo en la segunda ley de Newton sabemos que la llamada "Ley del Impulso" tiene la forma :

$$
\mathrm{F} d t=m d \mathrm{~V}
$$

Al miembro de la izquierda se le llama impulso y al de la derecha cantidad de movimiento o momentum.

Análogamente se define la cantidad de movimiento angular como el par producido por una partícula de masa $m$ que se desplaza en una trayectoria curva con un radio vector de giro $r$. En estas condiciones la Ley del Impulso Angular tiene la forma

$$
F \cdot r \cdot d t=m d V \cdot r=m\left(V_{2} r_{2}-V_{1} r_{1}\right)
$$

Ahora bien, si el vórtice ya está formado y no hay ninguna evidencia de agentes externos que lo hayan provocado, decimos que se trata precisamente de un vórtice libre. Si además se ignora la fricción o cualquier otra fuerza externa que afecte su movimiento, el par F.r. es nulo, debido a que las partículas no requieren ninguna fuerza para mantener su movimiento. Esto quiere decir, que la expresión (5) puede escribirse:

$$
m V_{2} r_{2}-m V_{1} r_{1}=0
$$

O, lo que es lo mismo:

$$
m V_{2} r_{2}=m V_{1} r_{1}=m V r=C t e
$$

Lo que equivale a decir que:

$$
\mathrm{Vr}=\mathrm{C}
$$

(V es la velocidad tangencial de la partícula)

La expresión (6) es la ley del vórtice libre. Ahora, analicemos la rotacionalidad de este fenómeno.

Como en el caso anterior y en cualquier momento, para el plano X - Y, se cumplen las expresiones (4), a saber:

$$
u=-V \frac{y}{r} \quad \text { y } \quad v=V \frac{x}{r}
$$

Y de acuerdo con Schlag (1957), en el vórtice libre deben cumplirse las condiciones:

$$
u=-C \frac{y}{r^{2}}=-C \frac{y}{x^{2}+y^{2}}
$$

Y,

$$
v=\mathrm{C} \frac{x}{r^{2}}=\mathrm{C} \frac{x}{x^{2}+y^{2}}
$$

Derivando ahora estas expresiones con respecto a las variables $Y$ y $X$, respectivamente, se tiene:

$$
\begin{gathered}
\frac{\partial u}{\partial y}=-C \frac{x^{2}+y^{2}-2 y^{2}}{\left(x^{2}+y^{2}\right)^{2}}=-C \frac{x^{2}-y^{2}}{r^{4}}=C \frac{y^{2}-x^{2}}{r^{4}} \\
\frac{\partial v}{\partial x}=C \frac{x^{2}+y^{2}-2 x^{2}}{\left(x^{2}+y^{2}\right)^{2}}=C \frac{y^{2}-x^{2}}{r^{4}}
\end{gathered}
$$

Por lo que:

$$
\frac{\partial v}{\partial x}-\frac{\partial u}{\partial y}=0
$$

Es decir, $\omega_{z}=0$, según la expresión (2-a). Análogamente, puede concluirse que en un vórtice libre $\omega_{x}=\omega_{y}=$ 0 . Es decir, el vector torbellino es nulo y esto significa que el vórtice libre es irrotacional, al contrario del vórtice forzado. Recuérdese que esto significa que las partículas se desplazan en las trayectorias curvas (en este caso), pero no giran con respecto a un eje perpendicular a dichas trayectorias, sino que siempre mantienen la misma posición con respecto a la línea que describe su movimiento. Desde luego, la expresión (7) garantiza que la vorticidad es: $\xi=0$ según Kolár (1983).

Por otra parte, obsérvese que la condición (6) le otorga al vórtice libre otra característica que consiste en que la velocidad tangencial de sus partículas es inversamente proporcional a su radio de giro. Esto exige que a una gran distancia del centro del vórtice (teóricamente infinita) la velocidad sería nula, lo que apoya la hipótesis de que el vórtice no se ha formado por efecto de algún agente superficial externo, sino que puede presentarse en un estanque en que el agua esté en completo estado de reposo.

La misma ley del vórtice libre explica también que si $\mathrm{r}=0$, la velocidad tangencial de la partícula sería infinita. Como esto no es posible, podemos garantizar que siempre habrá un pequeño radio $\mathrm{r} \neq 0$ en la salida del vórtice. La 
combinación del giro natural del vórtice libre con el flujo radial superficial hacia él, crea un movimiento espiral orientado en la dirección en que disminuye r. Este movimiento provoca que los cuerpos sólidos que se encuentran en la proximidad del vórtice, sean arrastrados en dicha dirección, situación que puede crear problemas serios en las turbinas de una central hidroeléctrica cuando el vórtice se forma en su bocatoma. Lo mismo sucede a los impulsores de las bombas cuando aparecen vórtices a la entrada de sus tuberías de succión. Por esta razón, en las estructuras mencionadas, el vórtice debe evitarse siempre, para ello, se observa que el efecto de succión se presenta sólo cuando la toma no está suficientemente sumergida. Es decir, la determinación de la sumersión mínima de una toma es un factor de absoluta importancia para el proyectista.

\section{Conclusiones}

Los vórtices son un fenómeno natural que tienen especial importancia para el ingeniero civil porque aparecen frecuentemente en todas las obras hidráulicas e influyen notablemente en su comportamiento. Por este motivo, se considera que los fundamentos del fenómeno deberían ser conocidos, tanto por los estudiantes de ingeniería como por los profesionales. La teoría que aquí se presenta, pretende explicar con sencillez, las leyes a que están sometidos dichos vórtices. Esta teoría no sólo ha sido verificada, sino que el lector puede comprobarla por sí mismo sin grandes dificultades.

Uno de los fundamentos en que se apoya la mencionada teoría, es el conocimiento de la rotacionalidad o irrotacionalidad de un vórtice que es uno de los conceptos que tradicionalmente no se exponen de forma suficientemente clara. Es por ello que se hace una demos. tración de estas propiedades para lograr un mejor entendimiento de los dos principales tipos de vórtices.

La intención de este artículo es apoyar al ingeniero que se interese en entender el origen y comportamiento de los vórtices que observa constantemente en su práctica profesional, ya que como estudiante en la Universidad sólo entrará en contacto con el tema si cursó estudios de posgrado. Es deseable también llamar la atención de los estudiantes de licenciatura de la carrera de ingeniería civil sobre el tema, por tal razón, se explica utilizando fundamentos matemáticos a su alcance para que le puedan servir si desea profundizar en lo que aún presenta muchas facetas por investigar. Por último, se hace hincapié en la importancia que tiene para el proyectista, tomar en cuenta los efectos dañinos que puesucción den causar los vórtices al romperse la continuidad del flujo; por ejemplo, en las obras de toma o en las tuberías de de los sistemas de bombeo.

\section{Bibliografía}

Comolet R. (1963). Mécanique Expérimentale des Fluides. Mason et Cie, París.

Daugherty, Franzini y Finnemore. (1985). Fluid Mechanics with Engineering Applications. Mc-Graw Hill.

Kolár V. (1983). Hydraulika (en checo). SNTL, Praga.

Levi E. (1965). Mecánica de los Fluidos. Facultad de Ingeniería, UNAM, México.

Levi E. (1989). El agua según la ciencia. CONACYT, CASTELL, México.

Schlag A. (1957). Hidraulique Générale. Dunod, Paris.

Tipler-Paul A. (1985). Física. Reverté SA, Barcelona.

\section{Semblanza del autor}

Humberto Gardea-Villegas. Realizó sus estudios en la Facultad de Ingeniería UNAM, en donde obtuvo el grado como ingeniero civil en 1961 y como maestro en hidráulica en 1965. Durante los años de 1962 y 1963, se le otorgó una beca para realizar investigación sobre modelos aerodinámicos en el Instituto de Investigaciones Hidráulicas de Praga, en la actual República Checa. A su regreso, desarrolló su tesis de maestría sobre la investigación que realizó en aquél país. Actualmente es profesor de la Facultad de Ingeniería en el área de hidráulica. Sus actividades no académicas se han desarrollado principalmente en la Comisión Federal de Electricidad, en la Secretaría de Recursos Hidráulicos y la Secretaría de Obras Públicas. Es autor de dos libros: Aprovechamientos hidroeléctricos y de bombeo e Hidráulica de canales, uno editado por Trillas en 1992 y el otro por la Fundación ICA, Facultad de Ingeniería, UNAM. 
